\title{
Passivity-based Stability in Explicit Force Control of Robots
}

\author{
Ribin Balachandran ${ }^{1}$, Mikael Jorda ${ }^{2}$, Jordi Artigas ${ }^{1}$, Jee-Hwan Ryu ${ }^{3}$ and Oussama Khatib ${ }^{2}$
}

\begin{abstract}
Direct force control of robots is challenging, particularly since the interaction with the environment can render the robot unstable. This paper presents the results of novel approaches for passivity-based stability for a particular direct force control method, namely explicit force control. A step-by-step procedure to passivate and stabilise the control loop is presented and it explains how Time Domain Passivity Approach, a passivity-based tool widely used in teleoperation and haptics has been extended and applied in explicit force control. The electrical circuit and network-port representations derived in the process allows the analytical evaluation of the system and can be applied in other control architectures as well. The stability methods are presented both qualitatively and quantitatively with simulations and hardware experiments. A discussion about the results obtained and the energy behavior is also provided. Results are promising and suggest that these methods can be used for stable and high-bandwidth force control of robotic manipulators.
\end{abstract}

\section{INTRODUCTION}

Many robotics tasks require a manipulator to be in contact with the environment. In the field of industrial robotics, for example, precise force control is required to perform certain tasks such as polishing, deburring and assembly of industrial components [1]. Different strategies to achieve force control have been extensively studied in the past, [2][5]. Today, two main approaches to control a manipulator in contact with environment are indirect force control and direct force control. Indirect force control regulates the dynamic relationship between the position and the force exerted by the robot with its environment without directly measuring the interaction forces with a sensor. Two common examples of indirect force control are impedance and stiffness controls. Direct force control on the other hand employs a feedback loop of the measured force.

In contrast to direct force control with inner motion loop (where the outer force loop commands a position to the inner position loop), explicit force control attempts to make the manipulator act as a pure force source, independently of the position and velocity of the manipulator. The advantages of this approach are faster reaction to environmental uncertainties and more precise force interaction. This strategy makes it ideal for tasks which require high bandwidth/frequency control of the robotic manipulator. An example of such a

\footnotetext{
${ }^{1}$ Ribin Balachandran and Dr. Jordi Artigas are with the department for Analysis and Control of Advanced Robotic Systems, German Aerospace Center (DLR), Germany ribin.balachandranedlr.de

${ }^{2}$ Mikael Jorda and Prof. Oussama Khatib (Director) are with the Robotics Lab, Stanford University, Stanford, USA. mjorda@stanford

${ }^{3}$ Prof. Jee-Hwan Ryu (Head of Department) is with the BioRobotics Laboratory of Korea University of Technology and Education, Cheonan, Korea. jhryuakoreatech.ac.in
}

task is the precise deburring of surfaces with sharp corners and edges.

Although stability of the controller is a common issue that appears for all these strategies, explicit force controllers are more vulnerable to instabilities due to the absence of inner motion loops. The stability of force controllers has been extensively studied in the past. Researchers suggest several possible causes, some of them being:

- Sensor dynamics

- Non-collocated actuator-sensor pairs

- Actuator bandwidth limits

- Effects of sampling and quantisation

- Filtering and delay

These factors causing instability have been studied both theoretically and experimentally. Dynamics effects are studied in detail in [6]-[8], while analysis about required bandwidth to get stability can be found in [7], [9]. The effects of kinematic and dynamic uncertainties in force control stability have also been studied in [10], [11]. A category of controllers that have been extensively studied and used to handle stability and performance issues are adaptive controllers [12], [13]. In [14], an adaptive control method is used for stabilising explicit force control by learning the model errors, and compensating for the feedback errors and even the delay. However, this method requires a long learning process for every new environment which makes it difficult to use practically. To the best of authors' knowledge, there have been not many researches to overcome the stability issues of explicit force control of robots.

Passivity is a tool widely used for stability in control systems with uncertainties. Although it is more conservative than certain other stability criteria (for example, absolute stability), it does not rely on the precise knowledge of system parameters [15]. In haptics and teleoperation, a passivitybased method to ensure the stability of the system is the Time Domain Passivity Approach (TDPA, [16], [17]). The idea is to monitor the energy in the system with a passivity observer, and to ensure the passivity of the system with a dedicated passivity controller which is a time-varying damper. This method has been shown to work when dealing with communication delay [17] and with uncertain environments [18].

The non-optimal controller gains due to low stability margins in explicit force controllers restrict them to low bandwidth control. In this study, we propose to combine TDPA and adaptive methods for a force-controlled robotic manipulator. The focus is on explicit force control. Its energy behavior is analysed, and energy observers are used as triggers to make the system passive via adaptive compensators. The step-by-step procedure for the analysis and 
stabilisation is presented which provides a better insight to the system behavior. The intermediate steps of designing the electrical network and network-port representation of the control system can be applied to analyse other systems as well. Four different adaptive methods are presented in this work which give rise to different system behaviors. The effectiveness of the methods are presented in both simulation and hardware experiments. A discussion about the results obtained is presented which will give a better understanding of the energy behavior in the system.

\section{EXPlicit ForCE CONTROL}

As explained in the introduction, in explicit force control, the interaction of the robot with the environment is feedback controlled using the measured interaction forces. The block diagram of the controller is shown in Fig. 1. The controller

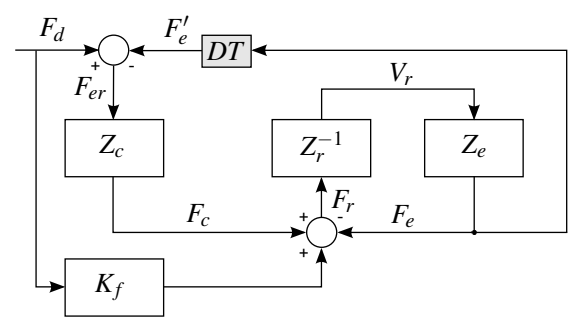

Fig. 1: Block diagram of a general explicit force control scheme.

$Z_{c}$ has input $F_{e r}$, the difference between the desired force $F_{d}$ and the measured force $F_{e}^{\prime}$. It is a general PID controller, the transfer function of which, in Laplace domain is given by:

$$
Z_{c}(s)=K_{p}+\frac{K_{i}}{s}+K_{d} s
$$

with $K_{p}, K_{i}$ and $K_{d}$ being the proportional, integral and derivative gains respectively and the resulting force being computed as:

$$
F_{c}(s)=\left(F_{d}(s)-F_{e}^{\prime}(s)\right)\left(K_{p}+\frac{K_{i}}{s}+K_{d} s\right) .
$$

This controller force is added to an optional feedforward of the desired force $K_{f} F_{d}$ before it is commanded to the robot with impedance $Z_{r}=s M_{r}+B_{r}$, which is assumed to be a single DoF mass and damper. The interaction between the robot and the environmental impedance (assumed to be a spring and damper with $Z_{e}=\frac{K_{w}}{s}+B_{w}$ ) produces force $F_{e}$. The resulting velocity of the robot is given by:

$$
V_{r}(s)=\frac{F_{c}(s)+K_{f} F_{d}(s)-F_{e}(s)}{Z_{r}(s)} .
$$

The block $D T$ represents practical deviations in the digital domain (from the real, physical force) including effects of discretisation, optional filtering, implicit delays and other non-collocation effects like link or joint flexibilities.

\section{ENERGY ANALYSIS}

Passivity of a system can be evaluated both analytically and experimentally. Analytical evaluation of passivity needs the precise models of the robot and environment which in case of interaction of robot with unstructured environments can be difficult. In the experimental evaluation, even without prior knowledge about the robot and environment, the power conjugated variables, force $f(t)$ and velocity $v(t)$ of a system can be measured and energy can be calculated by:

$$
E(t)=E(0)+\int_{0}^{t} f(\tau) v(\tau) d \tau
$$

where $E(0)$ is the initial energy stored in the system. Note that the $v$ is the velocity in the mechanical domain and should not be confused with voltage variables in the electrical domain. To ensure passivity of the system, the energy flowing out of the system $E^{\text {out }}(t)$ should be less than or equal to the sum of energy flowing into it $E^{i n}(t)$, and the initial energy storage $E(0)$. This, in mathematical terms, is:

$$
E(0)+E^{\text {in }}(t)-E^{\text {out }}(t) \geq 0,
$$

with $E^{\text {in }}$ and $E^{\text {out }}$ calculated with sign conventions as:

$$
\begin{gathered}
E^{\text {in }}(t)= \begin{cases}\int_{0}^{t} f(\tau) v(\tau) d \tau & \text { if } f(\tau) v(\tau)>0 \\
0 & \text { else. }\end{cases} \\
E^{\text {out }}(t)= \begin{cases}-\int_{0}^{t} f(\tau) \nu(\tau) d \tau & \text { if } f(\tau) v(\tau)<0 \\
0 & \text { else. }\end{cases}
\end{gathered}
$$

Time Domain Passivity Approach for haptics and teleoperation works exactly on these lines. In haptics, the energy of the system (the virtual environment represented by a 1port network) is identified and if the passivity rule in (5) is violated at any time, an additional damping is added into the system which modifies either the effort or the flow variable of the port. Similarly, in teleoperation, the energy flowing out from the communication channel (2-port network) at the master side ( $E^{\text {out }}$ ) is compared to the delayed in-flowing energy from the slave side ( $E^{\text {in }}$, and vice-versa), and additional damping is applied in a similar way. Detailed descriptions of the methods can be found in [16] and [17].

The stability methods proposed in this work for force control are direct extensions of TDPA. In order to analyse the energy behavior of the control system and ensure its passivity using TDPA, the block diagram of the control scheme in Fig. 1 is first converted into its electrical network representation. This conversion facilitates the straight forward derivation of the power correlated variables which are later required to make the network model of the system [19]. In order to proceed from initially having the block diagram of the controller to finally stabilising it, the following course of action is followed:

A. Design the electrical network representation of the control system.

B. Identify power correlated variables for each part of the control system.

C. Derive network-port representation using these variables.

D. Evaluate passivity for desired components of the network. 
E. Passivate these network elements using TDPA in case of observed activity.

These steps are explained in detail in the following sections.

\section{A. Electrical Analogy of the system}

The electrical network of the force control system is derived based on the mechanical-electrical analogy (forcevoltage:velocity-current). The equivalence of the two systems is also verified here.

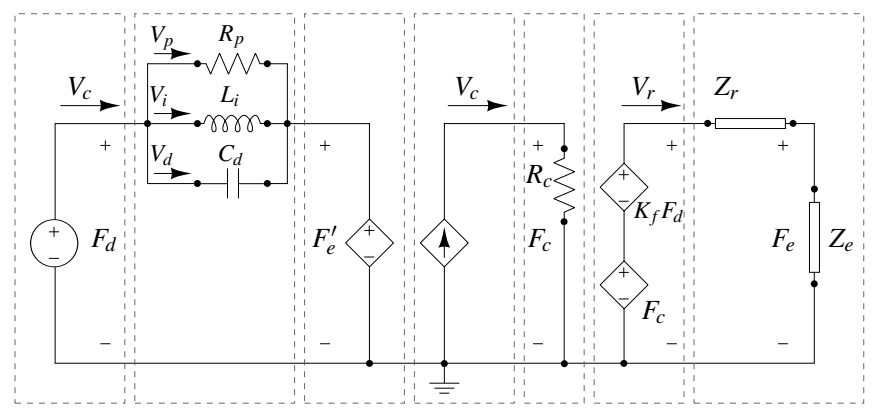

Fig. 2: Electrical network representation of explicit force control. Note that conventionally, independent sources are represented using circular symbols where as dependent sources are rhombus shaped. Note that the current source in the middle loop and the voltage source in the final loop are dependent on the corresponding quantities in the preceding loops.

As it can be seen in Fig. 2, the electrical analogy of the PID force controller has been designed as a combination of an RLC parallel circuit (first loop in Fig. 2) and a transimpedance circuit in (loop in the middle, which acts as a Current Controlled Voltage Source (CCVS)). The difference between the independent voltage source $F_{d}$ (desired force) and the dependent voltage source $F_{e}^{\prime}$ (measured force) with the RLC parallel load results in a total current $V_{c}$. Applying Kirchoff's circuit laws in the loop we get:

$$
\begin{aligned}
V_{p}(s) & =\frac{F_{d}(s)-F_{e}^{\prime}(s)}{R_{p}}, \\
V_{i}(s) & =\frac{F_{d}(s)-F_{e}^{\prime}(s)}{s L_{i}}, \\
V_{d}(s) & =\left(F_{d}(s)-F_{e}^{\prime}(s)\right) \times s C_{d}, \\
V_{c}(s) & =V_{p}(s)+V_{i}(s)+V_{d}(s), \\
& =\left(F_{d}(s)-F_{e}^{\prime}(s)\right)\left(\frac{1}{R_{p}}+\frac{1}{L_{i} s}+s C_{d}\right) .
\end{aligned}
$$

In the middle loop, the dependent current source $V_{c}$ produces a voltage $F_{c}$ across the resistance $R_{c}$, therefore from (8),

$F_{c}(s)=R_{c} \times V_{c}(s)=\left(F_{d}(s)-F_{e}^{\prime}(s)\right)\left(\frac{R_{c}}{R_{p}}+\frac{R_{c}}{L_{i} s}+s C_{d} R_{c}\right)$.

Now, comparing (9) with the (2),

$$
K_{p}=\frac{R_{c}}{R_{p}}, K_{i}=\frac{R_{c}}{L_{i}}, K_{d}=C_{d} R_{c} .
$$

The computed voltage $F_{c}$ is applied in the third loop along with the feedforward term $K_{f} F d$ resulting in a current $V_{r}$ given by,

$$
V_{r}(s)=\frac{F_{c}(s)+K_{f} F_{d}(s)-F_{e}(s)}{Z_{r}(s)},
$$

where $F_{e}$ is the voltage drop across the impedance $Z_{e}$ which is $F_{e}=Z_{e} V_{r}$ (the force produced due to the interaction between the robot and the environment). The value of the current in (11) is equivalent to the robot velocity explained in (3)

\section{B. Power Correlated Variables}

The benefit of deriving the electrical network of the system is that it makes it easier to identify the power correlated variables at different parts which is otherwise difficult to identify directly from the block diagram, [19]. As it can be seen in Fig. 2, the circuit components in each of the dashed boxes can be converted into a network with corresponding power variables. As an example, consider the leftmost dashed box. The current flowing out (flow variable) is $V_{c}$ and the voltage (effort) is $F_{d}$. Thus the power correlated pair for this port (box) is $\left(F_{d}, V_{c}\right)$.

\section{Network-Port Representation}

From the electrical circuit, the port representation of the system (Fig. 3) is directly derived where each of the dashed boxes in the circuit is converted into a network (both 1port and 2-port) with the corresponding power variables. The energy flowing in and out of the port can now be calculated from the power variables as explained in (7). An added benefit of finding the network model of any

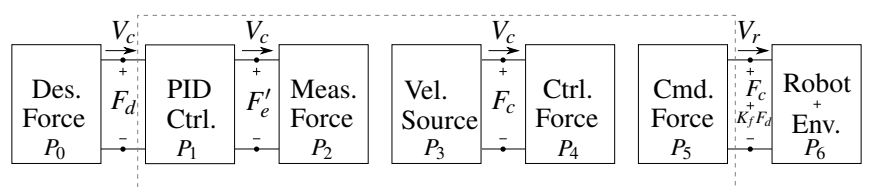

Fig. 3: Network representation of direct force control derived from the electrical network

system is that since the variables across all the ports are available, analytical evaluation of the system can easily be implemented by deriving its network parameters, for example, hybrid/scattering parameters.

\section{2-Port Network Passivity Evaluation}

As it has been long researched, there are several reasons why a force controller gets unstable. Any non-collocation between the commanded and measured forces might lead to instability. Discretisation and quantisation are other causes. From the network in (Fig. 3), it can be seen that all these factors take effect within the ports from $P_{1}$ to $P_{5}$. For example, a delay between commanded force and measured force is between $P_{5}$ and $P_{2}$. The discretisation effect of the controller is in $P_{1} . P_{0}$ is the desired force source to the loop and $P_{6}$ are the physical, mechanical components of robot and the environment, i.e., the real world, which are generally 
assumed to be passive. So, if ports $P_{1}$ to $P_{5}$ can be passivated, the whole series behaves as a passive system. Therefore, the chain of ports from $P_{1}$ to $P_{5}$ has been contained into a single port, namely, $P_{1-5}$, as shown in Fig. 4 . Now for the passivity

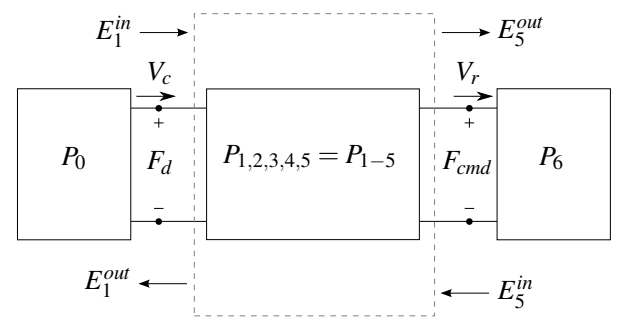

Fig. 4: Reduced form of the 2-Port representation showing input and output energies

analysis of the 2-port network $P_{1-5}$, the energy flowing in out of the system on both ports are calculated as shown in (7) with power correlated variables $\left(F_{d}, V_{c}\right)$ and $\left(F_{c m d}, V_{r}\right)$, where $F_{c m d}=F_{c}+K_{f} F_{d}$ is the force commanded to the robot. If we assume that the initial energy storage $E(0)$ of the system is zero, the passivity condition in (5) becomes,

$$
\begin{aligned}
E_{1-5}^{\text {in }}(t) & \geq E_{1-5}^{\text {out }}(t), \\
E_{1}^{\text {in }}(t)+E_{5}^{\text {in }}(t) & \geq E_{1}^{\text {out }}(t)+E_{5}^{\text {out }}(t),
\end{aligned}
$$

where $E_{1-5}^{\text {in }}$ and $E_{1-5}^{\text {out }}$ are the total energy flow into and out of the network respectively. Passivity of the system depends on the system parameters and it is always not guaranteed to be violated even in presence of the destabilising factors. Therefore, if the total energy is considered for checking passivity as shown in (12), the passivity of one direction (for example, energy flowing from $P_{1}$ to $P_{5}$ ), can hide the activity in the other direction (from $P_{5}$ to $P_{1}$ ). This could lead to instabilities. Therefore the passivity conditions in this work consider the individual flow directions, i.e.,

$$
\begin{aligned}
& E_{1}^{\text {in }}(t) \geq E_{5}^{\text {out }}(t), \\
& E_{5}^{\text {in }}(t) \geq E_{1}^{\text {out }}(t) .
\end{aligned}
$$

Although it is more conservative, it prevents the system from energy accumulation issues which lead to undesired behavior.

\section{E. Applying TDPA to ensure Passivity: Method 1}

The final step in the process explains how TDPA is applied here to passivate the system using the Passivity Observer (PO) and Passivity Controller (PC) concepts [17]. If PO observes any activity in one of the directions explained in (13), an additional damping, the PC is applied to dissipate this extra amount of active energy. As an example, the direction from port $P_{5}$ to $P_{1}$ is considered in the following equations for the conventional force modifying (impedance type) PC. This is done by modifying the feedback force $F_{e}^{\prime}$. The modified electrical network is shown in Fig. 5. In the discrete controller, with the current $V_{c}$ in the loop, the observed energy $E_{o b s}$ is given by,

$$
\begin{aligned}
E_{\text {obs }}(n)= & E_{5}^{\text {in }}(n)-E_{1}^{\text {out }}(n) \\
& +R_{p c}(n-1) V_{c}(n-1)^{2} \Delta T,
\end{aligned}
$$

where $\Delta T$ is the sampling time of the controller and $R_{p c}$ is the resistance required to be added into the loop to make it passive which is derived as:

$$
R_{p c}(n)= \begin{cases}-E_{o b s}(n) / \Delta T V_{c}(n)^{2} & \text { if } E_{o b s}(n)<0 \\ 0 & \text { else. }\end{cases}
$$

With this additional resistance, the feedback force to the controller becomes $F_{e}^{\prime}(n)+F_{p c}$, where

$$
F_{p c}(n)=V_{c}(n) R_{p c}(n) .
$$

The corrected output energy then becomes:

$$
E^{\text {corr }}(n)=E_{1}^{\text {out }}(n)-\sum_{0}^{n} F_{p c}(n) V_{c}(n) \Delta T .
$$

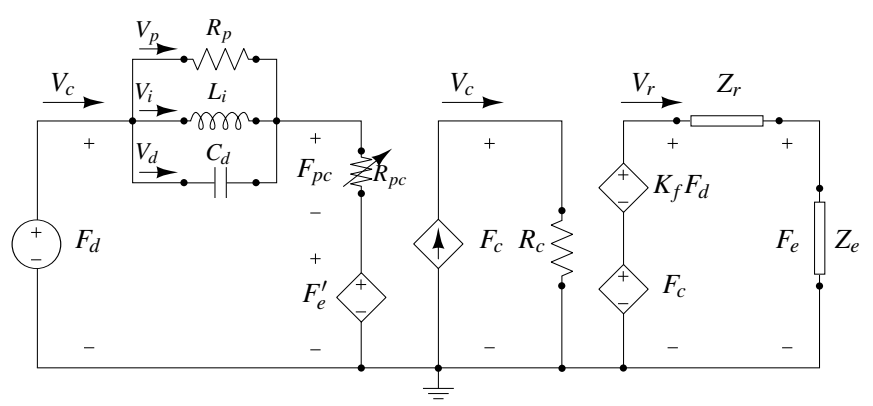

Fig. 5: Electrical network representation of direct force control with conventional force modifying PC: Method 1

\section{Proposed Control Strategies}

In the electrical domain, the $\mathrm{PC}$ is equivalent to adding time-varying resistors in the circuit. Another benefit of having the electrical analogy of the system is that this resistor can be placed at different parts of the circuit by rightly identifying the currents and voltages that need to be considered for the PO-PC approach. When we add resistors at different parts in the system, we get different behaviors, varying from conventional force and current modifying PCs to adaptive controller gains modification. The different possible methods are explained in this section.

\section{Method 2: Conventional Velocity Modifying PC}

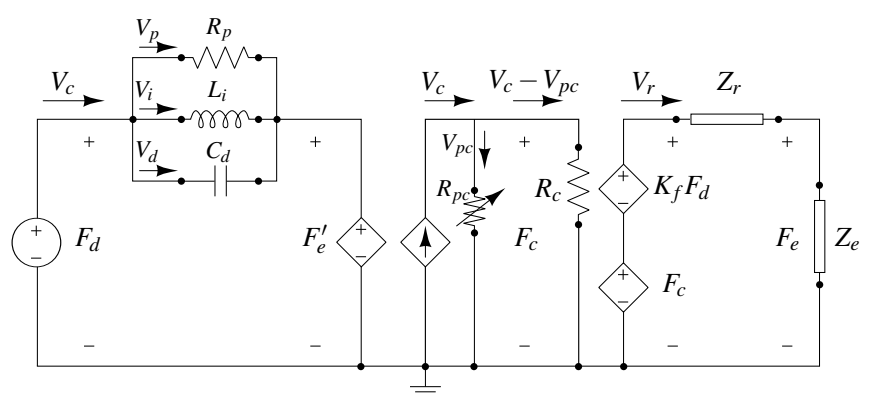

Fig. 6: Electrical network representation of direct force control with conventional current modifying PC

If the resistance is added parallel to $R_{c}$ in the transimpedance circuit as shown in Fig. 6, the resultant circuit 
modifies the energy of the system by modifying the current $V_{c}$ that flows through the resistance $R_{c}$ and thereby reducing the commanded forces as per (9). The equation for the current modification is also explained in [16]. With the observed energy in this case given by,

$$
E_{o b s}(n)=E_{5}^{i n}(n)-E_{1}^{o u t}(n)+\frac{F_{c}(n-1)^{2} \Delta T}{R_{p c}(n-1)},
$$

where $R_{p c}$ is the resistance added in the circuit in parallel with $R_{c}$. It is calculated as:

$$
\frac{1}{R_{p c}(n)}= \begin{cases}-E_{o b s}(n) / \Delta T F_{c}(n-1)^{2} & \text { if } E_{o b s}(n)<0 \\ 0 & \text { else. }\end{cases}
$$

The current $I_{p c}$ through this resistance and output voltage $F_{c}$ across $R_{c}$ which is then commanded to the robot respectively are,

$$
\begin{aligned}
I_{p c}(n) & =\frac{F_{c}(n-1)}{R_{p c}(n)} \\
F_{c}(n) & =R c\left[\left(F_{d}(n)-F_{e}^{\prime}(n)\right)\left(\frac{1}{R_{p}}+\frac{1}{L_{i} S}+s C_{d}\right)-I_{p c}(n)\right] .
\end{aligned}
$$

\section{Method 3: Adaptive tuning of the Controller Gains}

An interesting feature of modifying the value of the transimpedance resistor $R_{c}$ (as shown in Fig. 7) is that it indirectly modifies the controller gains so as to make the system passive. It has to be noted that in this case, the extra energy is removed from the network not by adding resistance, but by reducing the resistance of the transimpedance loop in the middle. This results in a reduction of the force commanded to the robot and thereby stabilises the system. Since it is a force modifying PC, the equations are as explained in (14), (15) and (16), replacing $F_{e}^{\prime}$ with $F_{c}$. The additional resistance $R_{p c}$ is removed from $R_{c}$ which reduces the commanded force $F_{c}$ to the robot given by:

$$
\begin{aligned}
F_{c}(n)= & \left(F_{d}(n)-F_{e}^{\prime}(n)\right)[\Phi(n)], \\
\Phi(n)= & \frac{R_{c}-R_{p c}(n)}{R_{p}} \\
& +\frac{R_{c}-R_{p c}(n)}{L_{i} s}+s C_{d}\left(R_{c}-R_{p c}(n)\right) .
\end{aligned}
$$

Now, comparing (23) with the (2),

$$
\begin{aligned}
K_{p}(n) & =\frac{R_{c}-R_{p c}(n)}{R_{p}}, \\
K_{i}(n) & =\frac{R_{c}-R_{p c}(n)}{L_{i}}, \\
K_{d}(n) & =C_{d}\left(R_{c}-R_{p c}(n)\right)
\end{aligned}
$$

It should be noted that $R_{p c}$ has to be upper-bounded by $R_{c}$ since otherwise, the system gets negative gains which will lead to instability.

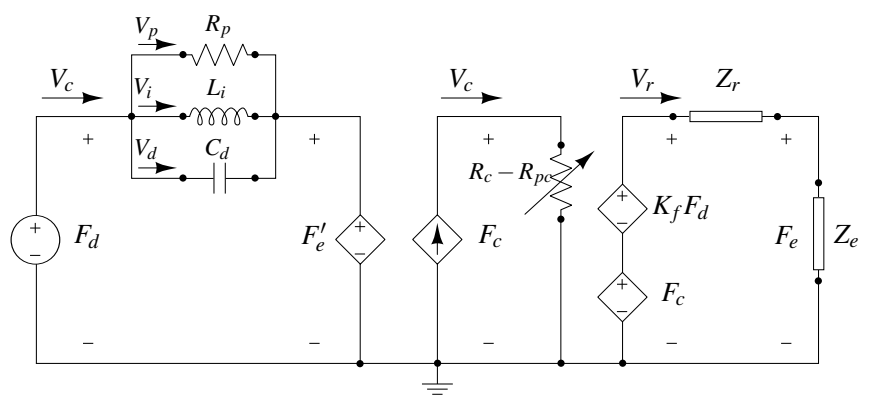

Fig. 7: Electrical network representation of direct force control with adaptable PID controller

\section{Method 4: Adaptive tuning of the Proportional Gain of the Controller}

If instead, the resistance $R_{p c}$ is added to the controller resistance $R_{p}$, it gives rise to a situation where the proportional gain of the controller can be tuned depending on the energy behavior of the system. The modified electrical network is shown in Fig. 8. It should be noted that the current flowing through the resistance $R_{p}$ is $V_{p}$ and this current has to be considered in (14), (15) for the calculations. It should be noted that varying the resistance $R_{p}$ modifies the controller current $V_{c}$ and eventually, the commanded voltage $F_{c}$ to the robot. The voltage $F_{c}$ is then given by:

$F_{c}=R_{c} \times V_{c}=\left(F_{d}-F_{e}^{\prime}\right)\left(\frac{R_{c}}{R_{p}(n)+R_{p c}(n)}+\frac{R_{c}}{L_{i} s}+s C_{d} R_{c}\right)$.

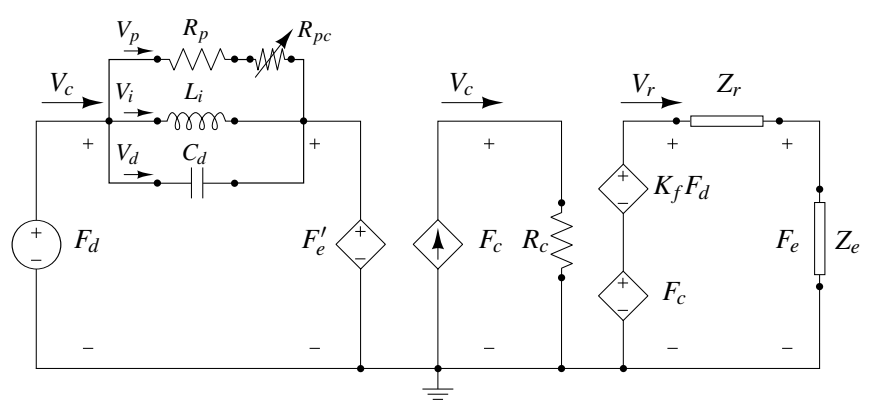

Fig. 8: Electrical network representation of direct force control with adaptable proportional gain

\section{Qualitative Analysis}

This section gives a qualitative analysis of the energy behavior in order to give the reader a physical interpretation. The case of a force controlled robotic manipulator's interaction with the environment during hard contacts is taken as an example. The robot is commanded with a force $F_{c m d}$. During hard contacts, the motion of the robot is stopped (or impeded) which means that its velocity $V_{r}$ is reduced. The power produced $\left(F_{c m d} V_{r}\right)$ during contact is really low due to the low robot velocity. As a result of the impact, if the robot is pushed back from the environment, a certain amount of negative power flows into the considered port $P_{5}$ since the commanded force and the robot velocity have 
different signs. The opposite signs are due to the fact that in a discrete system, the controller takes at least one sampling cycle to correct the commanded force in order to react to the impact. The interaction force measured $F_{e}^{\prime}$ is fed back to the controller. If the impact force is larger than the desired force $F_{d}$ (as it is during most impacts), the velocity output of the controller $V_{c}$ will have an opposite sign compared to the desired force. This results in a negative power output from port $P_{1}$. In a discrete system and a robot with inertia, if the energy output at $P_{1}$ due to increase in $F_{e}^{\prime}$ is larger than the energy input at $P_{5}$ due to the change in $V_{r}$, the system becomes active. Just by limiting the energy output by varying either $F_{e}^{\prime}$ or $V_{c}$, this output energy flow is limited to the input, thereby making the system passive.

\section{Results}

Experiments in both hardware and software simulation are implemented. The results of one series of force control parameters and interaction scenarios are shown for both simulation and hardware. Due to space constraints, the results of only one of the proposed strategies have been illustrated here, namely, that of Method. 3 from Sec. IV.

\section{A. Simulation}

Simulation experiments to study the energy behavior for the explicit force control of point mass of $1 \mathrm{Kg}$ and physical damping of $0.1 \mathrm{Ns} / \mathrm{m}$ are done in Matlab-Simulink. This point mass interacts with a virtual wall (implemented as a stiffness and damping) on both directions. In order to verify that passivity-based methods explained in this work are suitable for high bandwidth force demands in significantly unstructured environments, simulation results are shown for a time-varying sinusoidal input for the desired force of amplitude $5 \mathrm{~N}$ (whose frequency varies from $0.1 \mathrm{~Hz}$ to $10 \mathrm{~Hz}$ over a period of 20 seconds). The stiffness of the environment also varies with time (sinusoid of frequency $3 \mathrm{rad} / \mathrm{s}$ ) as shown in Fig. 9. The reason for instability is a single delay of $1 \mathrm{~ms}$ in the force measurement. Fig. 10 shows the controller without any passivity-based method and it can be seen that the system gets unstable with time and input frequency.

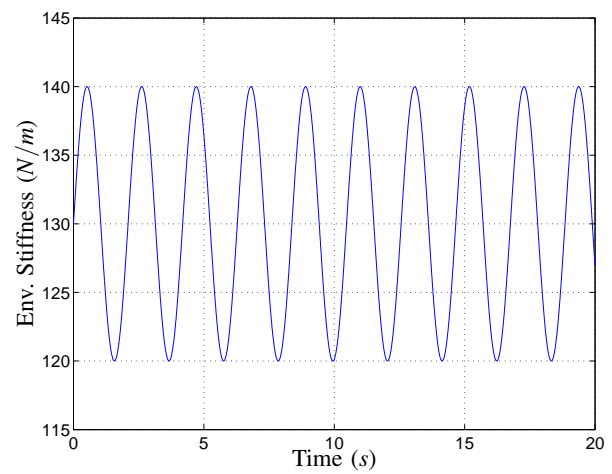

Fig. 9: Time varying stiffness of the environment

Fig. 11 shows the position and force following of the mass with Method. 3 from Sec. IV. The adaptive modification of the proportional gain of the controller is shown in Fig. 12.
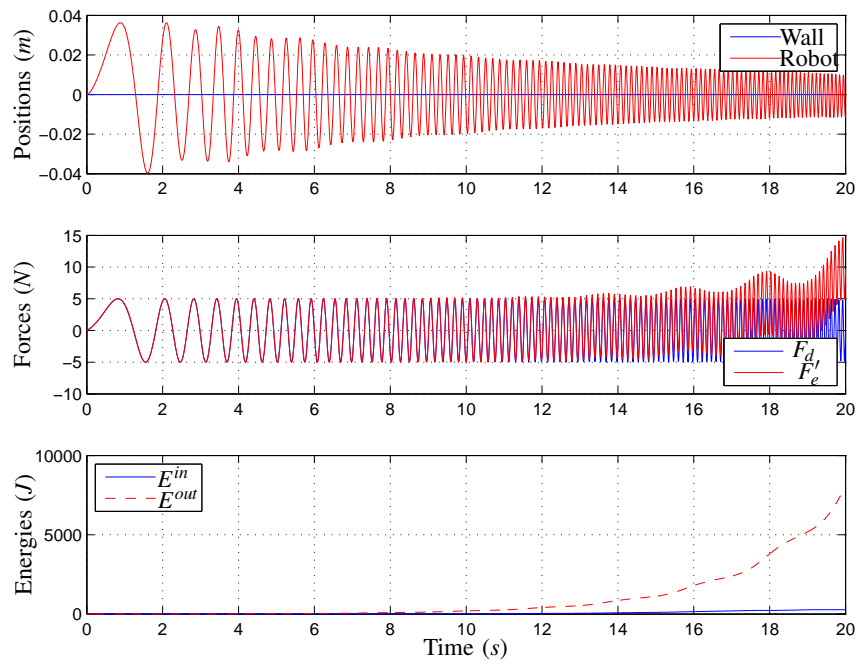

Fig. 10: Simulation system without any of the mentioned PCs which goes unstable.
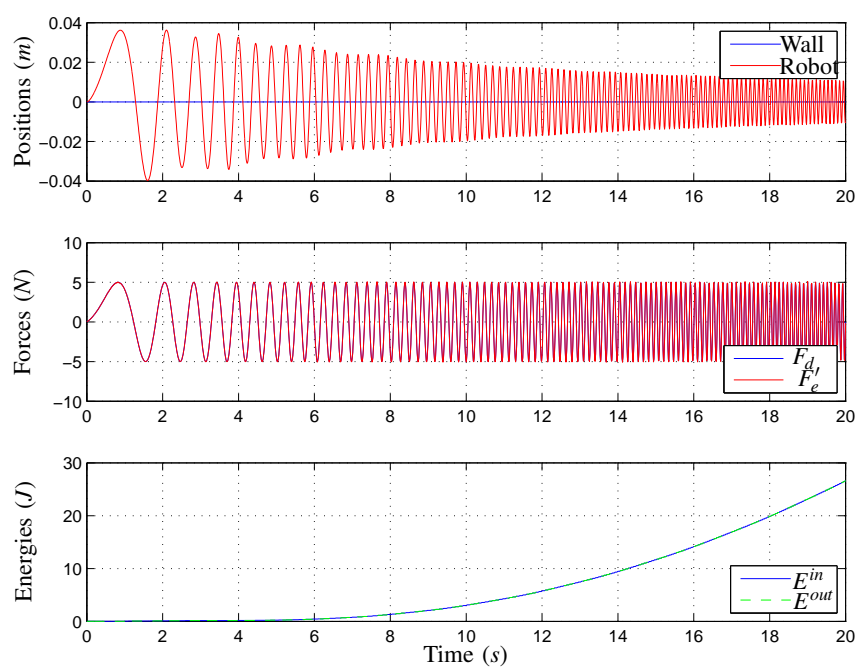

Fig. 11: Stable simulation with adaptive tuning of controller gains.

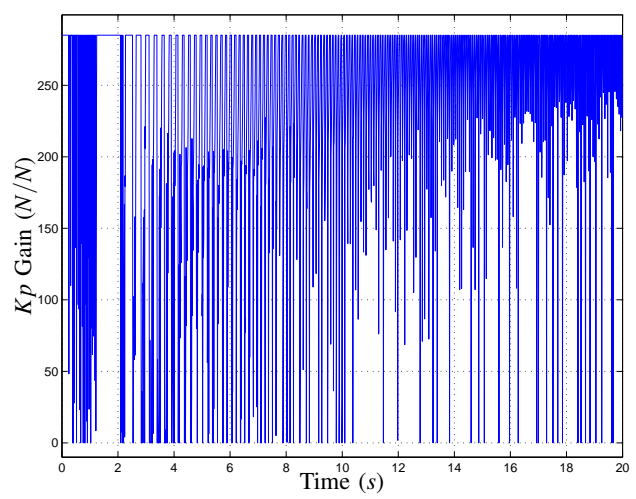

Fig. 12: Adaptive tuning of the controller gain using PC. It should be noted that the resulting high frequency force cannot be commanded directly to the robot. A solution to this problem has been given in the following discussion section. 


\section{B. Hardware Experiments}

Hardware tests were implemented on a single degree of freedom rotational actuator (Master-slave system from SensoDrive [20]) with inertia of $0.0001 \mathrm{Kgm}^{2}$ and virtual damping of $0.005 \mathrm{Nms} / \mathrm{rad}$. The device is equipped with a strain gauge torque sensor (SIMEX2k5Nm, also from SensoDrive). In order to show the worst case practical scenario, tests with a desired force of $0.8 \mathrm{Nm}$ step input and hardware limit on the device are conducted. The factors that lead to instability in the hardware tests are the delay and usage of filters for the measured forces. The torque sensor is placed in the interface between the actuator and the link of the system which leads to sensor dynamics which is another reason for instability. Results without (Fig. 13 and Fig. 14) and with (Fig. 15 and Fig. 16) passivity-based methods are shown. The $K_{p}, K_{i}$ and $K_{d}$ gains of the controller for this test are $4 \mathrm{Nm} / \mathrm{Nm}, 3 \mathrm{Nm} / \mathrm{Nm}$ and $0.006 \mathrm{Nm} / \mathrm{Nm}$ respectively.
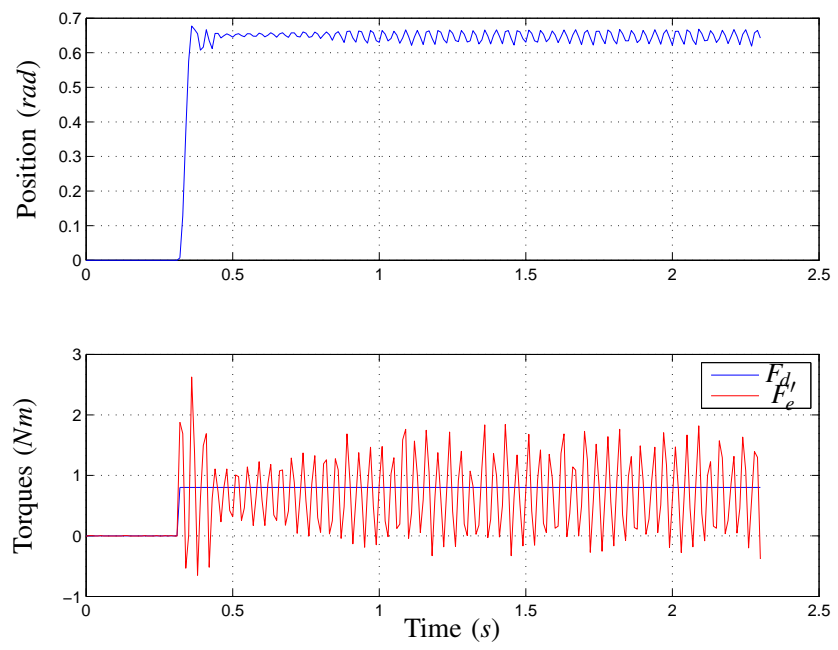

Fig. 13: Position and torques of the system during hard impact without PC
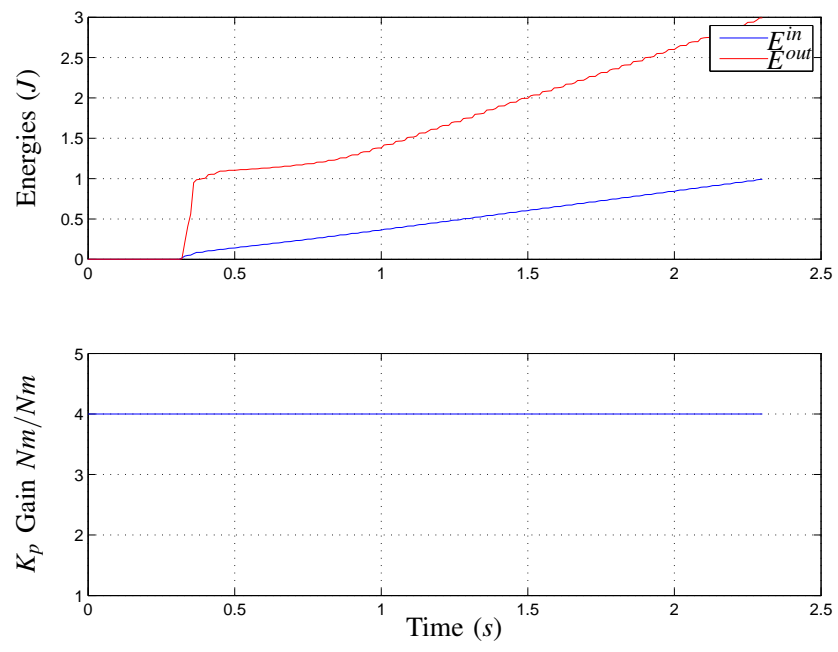

Fig. 14: Energy and gains of the system during hard impact without PC.
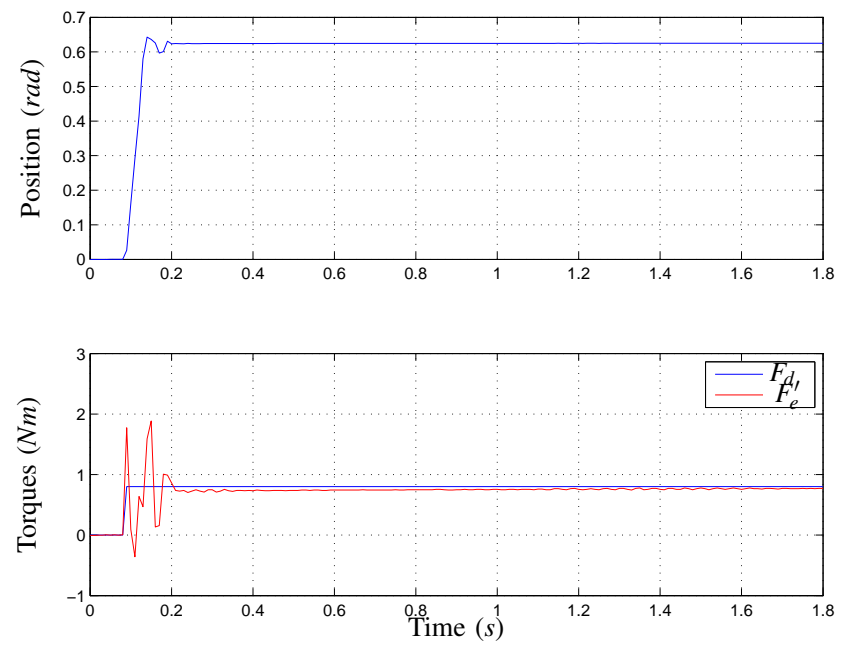

Fig. 15: Position and force following during hard impact with Method 3. The system is stable and the measured force settles to $94 \%$ of the desired value in $0.6 \mathrm{~s}$.
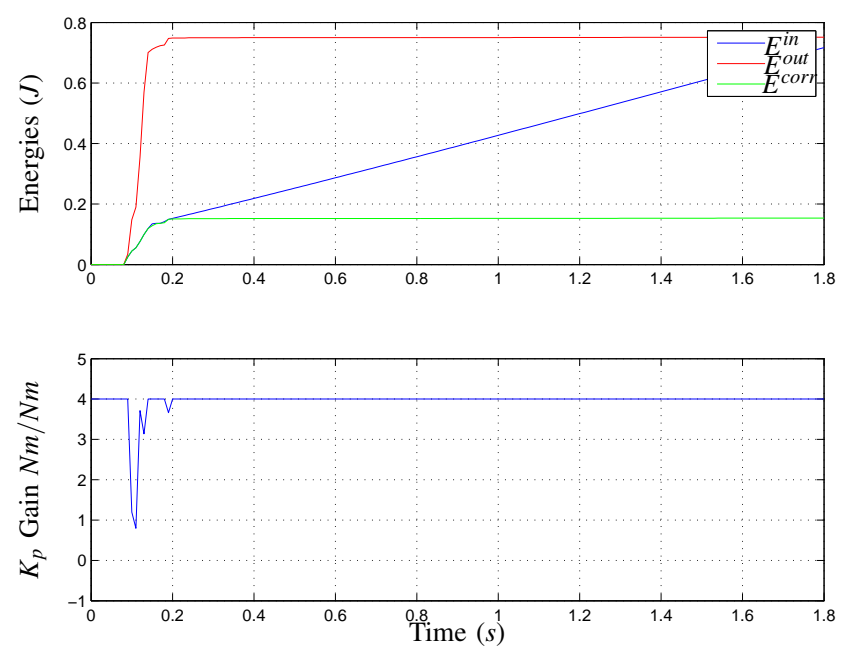

Fig. 16: Hard contacts with PC showing passivity and gain modification. It has to be noted that the green curve is the corrected output energy $E^{\text {corr }}$ defined in (17) (which unless corrected would be the red curve), based on the adaptive gain modification method. It is clear that the corrected output energy never gets larger that in input energy, which is the blue curve.

\section{DISCUSSION}

As it was evident from the results, the passivity-based controller ensures stability and also high bandwidth performance in explicit force control. As the gains are modified only when the passivity conditions are violated (as seen in Fig. 16), these strategies still offer high performance control. The authors also find limitations to these strategies. It can be seen that PC modifies the commanded forces to the robot at very high frequencies which would practically not 


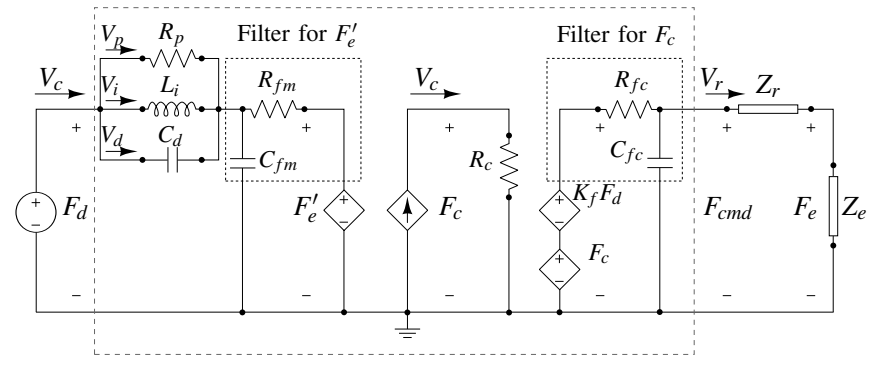

Fig. 17: Electrical Network Representation of a scheme with low-pass filters for measured and commanded forces

be possible to command to a robot. This high frequency force modification has been generally reported for TDPA as a short-coming. This is practically solved using low-pass filters. In real hardware, the noise in the raw force sensor data also makes it practically difficult to be used directly for which filters are used. But, it has been shown in [21] that usage of filters reduces the stability margins in force control. The passivity-based method proposed in this paper facilitates the usage of filters in both the measured forces and also the forces commanded to the robot. The filters for the commanded forces remove high frequency components introduced by the proposed adaptive methods. The reason for the stability in spite of the usage of filters is because both the filters can be designed to be inside the network considered for the energy analysis, and the system including the filters is made passive. The cut-off frequencies for both the filters are hardware specific and does not affect the passivity conditions due to the same reason. Fig. 17 shows the electrical network representation of the augmented system with filters, from which the network and passivity analysis can be done as explained in the paper.

\section{CONCLUSION AND FUTURE WORK}

Time Domain Passivity control has been extended to stabilise explicit force control for robots which otherwise is highly vulnerable to instabilities. A step-by-step procedure is explained with which not only force control of this architecture, but also other control systems could be passivated. The electrical to network-port formulation facilitates the use of different analytical tools for the system, as the network parameters become easily visible. The qualitative analysis provides the reader with a better insight to the energy behavior of the system. Four different strategies based on passivity and TDPA are presented. Simulation and hardware results show that these methods offer stable and high-bandwidth explicit force control in robotic manipulators. A possible future work is to analyse and compare the performances of these different strategies proposed in this paper. As the results presented are from the initial study validating the theory on a single DoF hardware, a natural extension of this work is to implement and evaluate the performance of the control strategies for the Cartesian force control of multi-DoF robotic manipulators.

\section{ACKNOWLEDGMENT}

This work is partially supported by the grant from the Stanford AI Lab-Toyota Center for Artificial Intelligence Research and the nuclear teleoperation project funded by the MOTIE KOREA. The support from Michael Panzirsch, Marco De Stefano (DLR, Germany) and Minkle Paul (Texas instruments, India) during the verification of the methods is highly appreciated and acknowledged.

\section{REFERENCES}

[1] B. Siciliano and L. Villani, Robot force control. Springer Science \& Business Media, 2012, vol. 540.

[2] D. E. Whitney, "Historical perspective and state of the art in robot force control," The International Journal of Robotics Research, vol. 6, no. 1 , pp. 3-14, 1987.

[3] G. Zeng and A. Hemami, "An overview of robot force control," Robotica, vol. 15, no. 05, pp. 473-482, 1997.

[4] R. Volpe and P. Khosla, "A theoretical and experimental investigation of explicit force control strategies for manipulators," IEEE Transactions on Automatic Control, vol. 38, no. 11, pp. 1634-1650, 1993.

[5] O. Khatib and J. Burdick, "Motion and force control of robot manipulators," Proceedings. 1986 IEEE International Conference on Robotics and Automation, vol. 3, no. 5, pp. 1381-1386, 1986.

[6] S. D. Eppinger and W. P. Seering, "On dynamic models of robot force control," 1986.

[7] S. D. Eppinger and W. P. Seering, "Three Dynamic Problems in Robot Force Control," IEEE Transactions on Robotics and Automation, vol. 8, no. 6, pp. 751-758, 1992.

[8] J. Hollerbach, "Dynamic stability issues in force control of manipulators," Proceedings. 1987 IEEE International Conference on Robotics and Automation, vol. 4, pp. 890-896, 1987.

[9] S. Eppinger and W. Seering, "Understanding bandwidth limitations in robot force control," in Robotics and Automation. Proceedings. 1987 IEEE International Conference on, vol. 4. IEEE, 1987, pp. 904-909.

[10] N. H. Mcclamroch and D. Wang, "Feedback Stabilization and Tracking of Constrained Robots," IEEE Transactions on Automatic Control, vol. 33, no. 5, pp. 419-426, 1988.

[11] C. C. Cheah, S. Kawamura, and S. Arimoto, "Stability of hybrid position and force control for robotic manipulator with kinematics and dynamics uncertainties," Automatica, vol. 39, pp. 847-855, 2003

[12] J.-J. Slotine and W. L. W. Li, "Adaptive strategies in constrained manipulation," Proceedings. 1987 IEEE International Conference on Robotics and Automation, vol. 4, pp. 595-601, 1987.

[13] R. Colbaugh, H. Seraji, and K. Glass, "Direct adaptive impedance control of robot manipulators," Journal of Robotic Systems, vol. 10, no. 2, pp. 217-248, 1993.

[14] Z. Luo, S. Fujii, Y. Saitoh, E. Muramatsu, and K. Watanabe, "Feedback-error learning for explicit force control of a robot manipulator interacting with unknown dynamic environment," in Robotics and Biomimetics, 2004. ROBIO 2004. IEEE International Conference on. IEEE, 2004, pp. 262-267.

[15] D. Lee and M. W. Spong, "Passive bilateral control of teleoperators under constant time-delay," IFAC Proceedings Volumes, vol. 38, no. 1, pp. 109-114, 2005

[16] B. Hannaford and J.-H. Ryu, "Time-domain passivity control of haptic interfaces," Robotics and Automation, IEEE Transactions on, vol. 18, no. 1, pp. 1-10, 2002.

[17] J.-H. Ryu, J. Artigas, and C. Preusche, "A passive bilateral control scheme for a teleoperator with time-varying communication delay," Mechatronics, vol. 20, no. 7, pp. 812-823, 2010.

[18] S. Kim, J. P. Kim, and J. Ryu, "Adaptive energy-bounding approach for robustly stable interaction control of impedance-controlled industrial robot with uncertain environments," IEEE/ASME Transactions on Mechatronics, vol. 19, no. 4, pp. 1195-1205, 2014.

[19] J. Artigas, J.-H. Ryu, C. Preusche, and G. Hirzinger, "Network representation and passivity of delayed teleoperation systems," in Intelligent Robots and Systems (IROS), 2011 IEEE/RSJ International Conference on. IEEE, 2011, pp. 177-183.

[20] "https://www.sensodrive.de/en/products/master-slave-system.php."

[21] C. H. An and J. M. Hollerbach, "Dynamic stability issues in force control of manipulators," in American Control Conference, 1987 IEEE, 1987, pp. 821-827. 